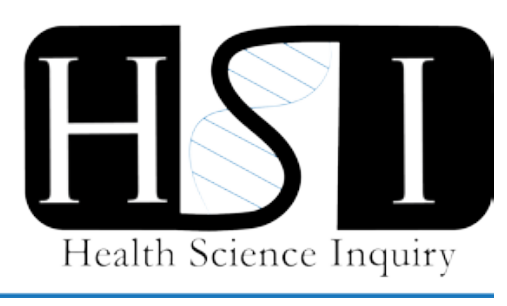

\title{
Childhood obesity: the importance of diet and physical activity \\ Meghan McGee
}

Department of Nutritional Sciences, University of Toronto

It is estimated that $14 \%$ of Canadian children are either overweight or obese [1]. These rates have remained relatively stable over the past few years and suggest a dire need for successful interventions. Obesity is a multifactorial disease characterized by increased caloric intake and decreased energy expenditure and is often accompanied by other chronic health conditions and serious economic impacts. Epidemiological evidence suggests overweight and obese children are $4-5$ times more likely to develop type 2 diabetes mellitus, 2-6 times more likely to develop hypertension and 2.4 times more likely to develop the metabolic syndrome in later life [2]. Moreover, it is estimated that excess weight and physical inactivity in Canada amount to annual economic burdens of $\$ 1$ billion and $\$ 1.5$ billion, respectively [3]. Several risk factors including poor dietary intake, marketing of energy-dense, nutrient-poor foods, physical inactivity and gut microbial composition are believed to play a role in the etiology of obesity. Furthermore, habit-forming behaviour begins in early childhood and tracks into adolescence and adulthood; thus promoting a healthy lifestyle in early childhood is crucial.

Dietary intake in early childhood is important to support energy requirements for appropriate growth and metabolism; however, many children overconsume calories leading to excessive weight gain, fat deposition and altered gut microbial composition. Children who consume a diet characterized by energy-dense, high-fat, low-fibre foods are more likely to develop obesity in later life and the impact of diet on microbial composition is believed to play a significant role [4]. In a recent study comparing fecal microbiota of European children to rural African children, European children typically consumed a highfat, low-fibre diet and had lower levels of Bacteroidetes and higher amounts of Firmicutes compared to African children consuming high-fibre, low-fat diets [5]. The ratio of Firmicutes to Bacteroidetes differs in lean and obese individuals, where greater amounts of Firmicutes and lower amounts of Bacteroidetes is associated with obesity [6]. In another study, obese adults were randomly assigned to either a low-fat or low-carbohydrate diet to be followed for one year [6]. Weight loss was observed in both groups and was correlated with an increased abundance of Bacteroidetes [6]. These bacteria have different genetic components, engage in different metabolic activities and appear modifiable through dietary intake. Therefore, gut microbial composition may partly explain the link between dietary intake and obesity.

The marketing of unhealthy foods to children is big business. Ultra-processed, calorie-dense foods are shamelessly promoted on children's television channels, video games and social media. In recent years, food and beverage television advertising to children has increased by $17 \%$ on all stations and by $5 \%$ on children-specific stations in Toronto [7]. A systematic review and metaanalysis of 17 studies found unhealthy dietary marketing to children was associated with increased caloric intake during or shortly following exposure and these children were $10 \%$ more likely to choose energy-dense, nutrientpoor foods [8]. Moreover, consumption of these types of foods stimulates several neural pathways involved in reward, which may induce addictive-like traits in food consumption, increase unhealthy eating behaviours such as binge eating and further contribute to the rising rates of overweightness and obesity [9]. Overall, these actions play into our evolutionary inclination towards energy-dense foods, making over-consumption easy and in line with normality.

In addition to consuming a healthy diet, physical activity is crucial for energy expenditure and weight management. However, many Canadian children fail to meet physical activity guidelines and, instead, spend several hours each day in sedentary behaviours [10]. A systematic review and meta-analysis using data from 7,351 youth across 5 studies found substituting sedentary time for moderate-to-vigorous intensity exercise significantly reduced body fat percentage by $2.5 \%$ [11]. When 60 minutes of sedentary behaviour was reallocated to moderate-to-vigorous physical activity, a $4.5 \%$ decrease in body fat percentage was observed [11]. Interestingly, reallocating sedentary time to light physical activity did not yield a significant effect on body composition. This suggests that moderate-to-vigorous physical activity should be promoted and prioritized in daily life, while efforts should be made to reduce time spent in sedentary behaviours. Finally, promoting physical activity 
in early childhood is important for instilling healthy lifestyle behaviours and reducing the risk for chronic disease later in life.

Childhood obesity is influenced by a wide array of interacting factors. Many are modifiable. Promoting healthy behaviours and limiting food and beverage advertising in early childhood is crucial for fostering positive attitudes towards a healthy diet and participation in physical activity. Not only do health-promoting behaviours reduce the risk for obesity and other chronic diseases, but they may improve children's gut microbial composition. It is time we act from a personal, public and political perspective to encourage the success of our children and their future.

\section{References}

[1] Rao, D. P., Kropac, E., Do, M. T., Roberts, K. C., Jayaraman, G. C. (2016) Childhood overweight and obesity trends in Canada. Health Promot Chronic Dis Prev Can Res Policy Pract., 36 (9), 194198.

[2] Pulgar'on, E. R. (2013) Childhood Obesity: A Review of Increased Risk for Physical and Psychological Comorbidities. Clin Ther., 35 (1), A1832.

[3] Krueger, H., Krueger, J., Koot, J. (2015) Variation across Canada in the economic burden attributable to excess weight, tobacco smoking and physical inactivity. Can J Public Health Rev Can Sante Publique. 106 (4), e171-177.

[4] Ambrosini, G. L. (2014) Childhood dietary patterns and later obesity: a review of the evidence. Proc Nutr Soc., 73 (1), 137146.
[5] Filippo, C. D., Cavalieri, D., Paola, M. D., Ramazzotti, M., Poullet, J. B., Massart, S., et al. (2010) Impact of diet in shaping gut microbiota revealed by a comparative study in children from Europe and rural Africa. Proc Natl Acad Sci., 107 (33), 1469114696.

[6] Ley, R. E., Turnbaugh, P. J., Klein, S., Gordon, J. I. (2006) Microbial ecology: Human gut microbes associated with obesity. Nature, 444 (7122), 10221023.

[7] Potvin Kent, M., Wanless, A. (2014) The influence of the Children's Food and Beverage Advertising Initiative: change in children's exposure to food advertising on television in Canada between 2006-2009. Int J Obes, 38 (4), 558562.

[8] Sadeghirad, B., Duhaney, T., Motaghipisheh, S., Campbell, N. R. C., Johnston, B. C. (2016) Influence of unhealthy food and beverage marketing on children's dietary intake and preference: a systematic review and meta-analysis of randomized trials. Obes Rev Off J Int Assoc Study Obes., 17 (10), 945959.

[9] Murray, S., Tulloch, A., Gold, M. S., Avena, N. M. (2014) Hormonal and neural mechanisms of food reward, eating behaviour and obesity. Nat Rev Endocrinol., 10 (9), 540552.

[10] Colley, R. C., Carson, V., Garriguet, D., Janssen, I., Roberts, K. C., Tremblay, M. S. (2017) Physical activity of Canadian children and youth, 2007 to 2015. Health Rep., 28 (10), 816.

[11] García-Hermoso, A., Saavedra, J. M., Ramírez-Vélez, R., Ekelund, U., Del Pozo-Cruz, B. (2017) Reallocating sedentary time to moderate-to-vigorous physical activity but not to light-intensity physical activity is effective to reduce adiposity among youths: a systematic review and meta-analysis. Obes Rev Off J Int Assoc Study Obes., 18 (9), 10881095.

Meghan McGee is a PhD candidate in the Department of Nutritional Sciences at the University of Toronto. Meghan is working on improving the health outcomes of children who were born preterm. She aims to understand the risk factors for childhood overweight and obesity and is investigating the role of donor breastmilk as a preventative measure in early life. Meghan completed her Bachelor's degree at McGill University and her Master's degree at the University of Ottawa. In addition to her PhD, Meghan is the founder of the epidemiological consulting firm, EpiConsult. 